\title{
ERRATUM
}

Yoko Kawai $\cdot$ Akihiko Moriyama $\cdot$ Kiyofumi Asai

Carrie M. Coleman-Campbell $\cdot$ Satoshi Sumi

Hideko Morishita • Mariko Suchi

\section{Molecular characterization of histidinemia: identification of four missense mutations in the histidase gene}

Published online: 30 September 2005

(C) Springer-Verlag 2005

\section{Hum Genet 116: 340-346}

Unfortunately, in Table 1 the forward and reverse primers were not divided correctly. The correct table is printed blow:

The online version of the original article can be found at http:// dx.doi.org/10.1007/s00439-004-1232-5

Y. Kawai

Pediatric Nursing, Nagoya City University School of Nursing,

Nagoya, Japan

A. Moriyama

Graduate School of Natural Sciences, Nagoya City University,

Nagoya, Japan

K. Asai

Department of Molecular Neurobiology,

Graduate School of Medical Sciences,

Nagoya City University, Nagoya, Japan

S. Sumi $\cdot$ H. Morishita $\cdot$ M. Suchi

Department of Pediatrics, Neonatology and Congenital Disorders, Graduate School of Medical Sciences, Nagoya City University,

Nagoya, Japan

C. M. Coleman-Campbell

The General Clinical Research Center,

The Children's Hospital of Philadelphia,

Philadelphia, PA, USA

M. Suchi

Department of Pathology and Laboratory Medicine,

The Children's Hospital of Philadelphia,

University of Pennsylvania School of Medicine,

Philadelphia, PA, USA

Present address: M. Suchi $(\bowtie)$

Department of Pathology, Children's Hospital of Wisconsin,

Medical College of Wisconsin, 9000 West Winconsin Avenue,

Milwaukee, WI 53226, USA

E-mail: msuchi@mail.mcw.edu

Tel.: + 1-414-2662526

Fax: + 1-414-2662779 


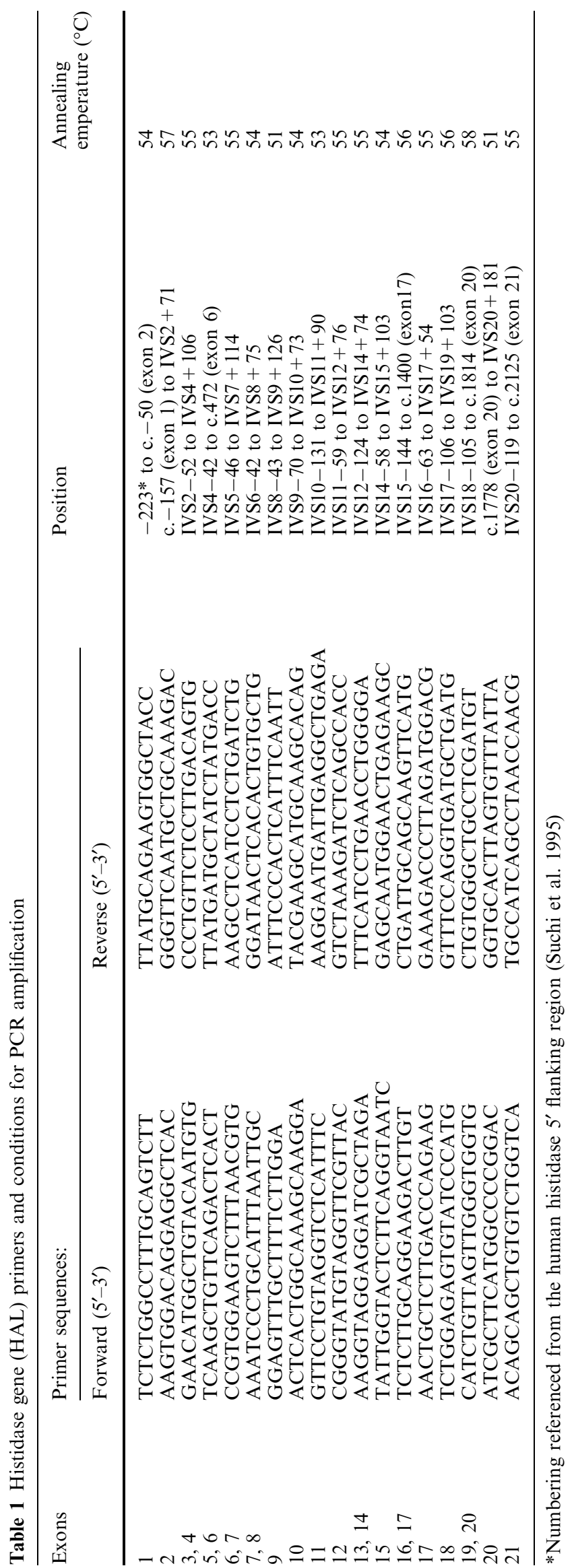

